\title{
Age- and Sex-Related Percentiles of Skinfold Thickness, Waist and Hip Circumference, Waist-to-Hip Ratio and Waist-to-Height Ratio: Results from a Population-Based Pediatric Cohort in Germany (LIFE Child)
}

\author{
Elisa Rönnecke ${ }^{a, b} \quad$ Mandy Vogel $^{a}$ Sarah Bussler ${ }^{a, b} \quad$ Nico Grafe ${ }^{a}$ \\ Anne Jurkutat $^{a} \quad$ Maike Schlingmann ${ }^{a}$ Antje Koerner ${ }^{a}$ b Wieland Kiess ${ }^{a}$, b \\ aLIFE Leipzig Research Center for Civilization Diseases, University of Leipzig, Leipzig, \\ Germany; ${ }^{b}$ Hospital for Children and Adolescents, Center of Pediatric Research, University \\ of Leipzig, Leipzig, Germany
}

\section{Keywords}

Adiposity - Skinfold thickness - Waist circumference - Fat distribution - Subcutaneous fat tissue $\cdot$ Children

\begin{abstract}
Background: Skinfold thickness (ST), waist circumference (WC) and hip circumference (HC) measurements are simple methods for assessing fat tissue at defined body parts. We examined these parameters in a cohort of healthy children and adolescents in Leipzig. Our study provides current percentile curves for biceps, triceps, subscapular and iliac crest ST, plus WC, HC, waist-to-hip ratio and waist-to-height ratio. Methods: 6,344 visits were recorded involving 2,363 individuals from 3 to 16 years in age. Continuous age- and gender-related percentiles (3rd, 10th, median, 90th, 97th percentiles) were estimated using Cole's LMS method. $\boldsymbol{R e}$ sults: For biceps and triceps ST, boys show a peak at the beginning of adolescence with a subsequent decrease, while percentile values among girls rise across the age range. Subscapular and iliac crest percentiles also show increasing curves with disproportionately high values for P90 and P97. Boys show higher values of WC, girls have higher levels of HC. WC and HC median percentiles constantly increase in both sexes with a plateau at the age of 16 for girls. Conclusion: Trends for all parameters of body fat are in line with other national and international studies. Unlike the KiGGS study, our study provides circumference data across the whole of our age range, i.e. from 3 to 16 years.


Rönnecke et al.: Age- and Sex-Related Percentiles of Skinfold Thickness, Waist and Hip Circumference, Waist-to-Hip Ratio and Waist-to-Height Ratio: Results from a

Population-Based Pediatric Cohort in Germany (LIFE Child)

\section{Introduction}

Germany has witnessed an increase in the prevalence of obesity and overweight in line with the global trend, and though levels have stabilized they remain worryingly high: $15.4 \%$ of German children aged 3-17 are overweight, including 5.9\% (of the total) who are obese [1]. Compared to the reference populations from 30 years ago, we observe a $50 \%$ increase in overweight in children [2]. It is well known that overweight in childhood is associated with overweight or obesity in adulthood, and there is a higher probability that such individuals will remain overweight, with the accompanying risk of comorbidities [3]. Furthermore, it constitutes a direct risk factor for metabolic syndrome [4]: in children, obesity - especially with high levels of visceral adipose tissue - affects the composition of serum metabolome [5] and is associated with increased intima-media thickness [6], higher lipid and blood pressure levels [7, 8], and a greater risk of developing coronary heart disease in later life [9]. Obesity is characterized by a pathologically increased body fat percentage (BFP) of the total body mass [10]. The BMI (in $\mathrm{kg} / \mathrm{m}^{2}$ ) is a recommended measure of body fat [11]; it is used to define overweight and obesity and thereby utilized as a predictor for cardiovascular risk [12]. Ageand sex-related BMI percentiles are used for children and adolescents with cutoffs for overweight ( $>90$ th percentile) and obesity ( $>97$ th percentile) [13]. The determination of body composition and BFP are highly relevant to assessing the validity of anthropometric measurements like the BMI in pediatrics, as BMI is calculated from weight and height measurements without considering BFP, which changes naturally during childhood and adolescence [14]. A BMI measurement may indicate a high weight rather than a high BFP [15], something that becomes particularly apparent in boys during puberty [16]. There are several methods to assess BFP: magnetic resonance imaging (MRI), which makes it possible to measure visceral as well as subcutaneous adipose tissue; dual-energy X-ray absorptiometry (DXA), which is still the gold standard in most studies; hydrodensitometry [17]; and ultrasound [18]. However, these examination methods are expensive, time-consuming, or involve radiation exposure, making them unsuitable for use in pediatric examinations or even as a screening instrument. Skinfold thickness (ST) is measured in the context of anthropometric surveys to assess the BFP indirectly. Measurements are done using a caliper on skinfolds at defined parts of the body, e.g., over the biceps or the triceps as well as subscapularly und suprailiacally. ST is assumed to measure the amount of subcutaneous fat tissue, which correlates with total body fat [19]. Various studies recommend ST measurements as the preferred screening tool in adolescents because ST predicts adult body fatness better than adolescent BMI and provides more accurate estimates of adiposity [20-22].

In Germany, several ST percentile curves from local studies are available, for instance those using representative samples from the populations of Jena [23], Saarland [24], and Nuremberg [25]. Using Slaughter's equations of BFP it is possible to estimate the BFP for the different age- and gender-related percentiles from the triceps and subscapular ST [25]. Kromeyer-Hauschild et al. [23] described a significant increase of BFP compared to data from children in 1975. Waist (WC) and hip circumferences (HC), waist-to-hip ratio (WHR), and waist-to-height ratio (WHtR) are further parameters for determining body composition and assessing risk factors. WC in children and adolescents can vary depending on age, sex and ethnic group [26], while HC is additionally influenced by bone structure (pelvis width) and muscle mass. HC is not commonly used as parameter in its own right, but rather for calculating indices such as WHR or WHtR. WHR (WC/HC) cutoff points are used to categorize the distribution of body fat in adults with the same BMI and help to define overweight in terms of "apple shape" or "pear shape." It is known that central obesity (apple-shape, high WHR) contributes a higher risk than general obesity [27]. However, to date there are no established cutoffs for clinical practice with children and adolescents [28], likewise with WHtR, which is 
Rönnecke et al.: Age- and Sex-Related Percentiles of Skinfold Thickness, Waist and Hip Circumference, Waist-to-Hip Ratio and Waist-to-Height Ratio: Results from a

Population-Based Pediatric Cohort in Germany (LIFE Child)

a more accurate tool for obesity diagnosis and a better prognosticator of associated health risks than BMI [28-30]. Circumference measurements are cheap and simple anthropometric methods, and both $\mathrm{HC}$ and WC are good predictors of intra-abdominal fat and cardiovascular risk factors [31-34]. Additionally, different pediatric studies have shown that BMI systematically underestimates the prevalence of obesity compared to the WC [35, 36]. WC may also identify normal-weight children and adolescents with cardiovascular risk factors [37, 38].

The different waist and hip parameters and their combination with the BMI are helpful in assessing the distribution of body fat, and thereby in assessing cardiovascular risk factors, especially during pubertal development [39]. In this paper, we provide current age- and sexrelated percentiles for triceps, biceps, subscapular and iliac crest ST as well as WC, HC, WHR and WHtR based on a large cohort of healthy children between 3 and 16 years of age from the LIFE child cohort. The present study aims to update information about gender-related distribution and development of fat tissue as an indicator for elevated BFP.

\section{Participants and Methods}

\section{Study Design}

The data were collected as part of the LIFE Child study in Leipzig, Germany. The LIFE Child study is a longitudinal population-based cohort study based at the Leipzig Research Center for Civilization Diseases (LIFE). The study aims to examine the environmental, genetic, and metabolic factors that influence the development of civilization diseases in children and adolescents $[40,41]$.

\section{Setting}

In accordance with German data protection law, data has been pseudonymized. Every visit to the study center includes clinical history and examination, anthropometry, blood collection, urine samples, and different age-dependent questionnaires and examinations. The children and their parents are asked to attend an annual follow-up appointment [41]. ST measurements are taken from age 2 upwards. Only cases with complete datasets of ST measurements, WC, and HC from the LIFE Child health cohort were included in the analysis.

\section{Participants and Variables}

The participants are primarily healthy (not suffering from any chromosomal or syndromic diseases) children and adolescents from Leipzig and its environs. The variables considered are: triceps, biceps, subscapular and iliac crest ST; WC and HC; and WHR, WHtR and BMI, with participants grouped according to sex and age.

\section{Data Sources/Measurements}

The measurements were taken during anthropometry assessment of the participants by trained and certified observers. The measurement of ST (calipometry) was taken using a "Holtain" (Holtain, Crosswell) or "Harpenden Skinfold" (Baty International, Burgess Hill) caliper with a dial graduation of $0.2 \mathrm{~mm}$. The ST was measured on the right-hand side of the body. Participants had an upright posture with relaxed arms. Triceps skinfold was measured over the midpoint of the triceps muscle between the olecranon process and the acromion. Biceps skinfold was measured over the midpoint of the biceps muscle between the olecranon process and the acromion. The subscapular skinfold was measured just below the right scapula, with the fold pinched to run at an angle of $45^{\circ}$ to the spine. The iliac crest skinfold was measured in the mid-axillary line parallel to the iliac crest. Every skinfold measurement was taken three times in succession, and the median of the three values was used. BMI was 
Rönnecke et al.: Age- and Sex-Related Percentiles of Skinfold Thickness, Waist and Hip Circumference, Waist-to-Hip Ratio and Waist-to-Height Ratio: Results from a

Population-Based Pediatric Cohort in Germany (LIFE Child)

calculated as body weight $(\mathrm{kg})$ divided by the square of the height $\left(\mathrm{m}^{2}\right)$. Body weight was measured in light underclothes to an accuracy of $50 \mathrm{~g}$ using a "Seca 701" calibrated electronic scale (Seca GmbH and Co. KG, Hamburg, Germany). Height was measured to an accuracy of $0.1 \mathrm{~cm}$ using a "Dr. Keller I" stadiometer (Längenmesstechnik GmbH Limbach, Limbach-Oberfrohna, Germany). Circumferences were measured using a "Picco" tape measure (Hoechstmass Balzer GmbH, Sulzbach, Germany). WC was measured as the smallest circumference between the rib cage and the superior border of the iliac crest at the end of expiration. HC was measured at the greater trochanters at the widest part of the hips. WHR was calculated as WC $(\mathrm{cm})$ divided by HC $(\mathrm{cm})$. WHtR was calculated as WC $(\mathrm{cm})$ divided by height $(\mathrm{cm})$. Socioeconomic status was determined using the "social class index" [42], which considers the educational level, income and professional status of the participants' parents. To calculate this index, a points system is used, and families are classified as having low level (3-8 points), middle level (9-14 points) or high level (15-21 points) social status. BFP (\%) was estimated using the BFP equations of Slaughter et al. [43] for white children and adolescents. The male participants are separated into age groups to offer analysis at different levels of maturity; the equations can be found in the supplementary material (available at http://content.karger. com/ProdukteDB/produkte.asp?doi=494767).

\section{Study Size}

6,344 cases with complete ST, HC and WC measurements were available $(2,980$ involving girls, 3,364 involving boys) representing 2,363 individuals (3-16 years, LIFE Child cohort) from 1,710 families. Due to the small number of subjects younger than 3 or older than 16 attending the examinations, the age range was reduced to 3 up to 16 years (16.99). The resulting data were used for the calculation of the percentile curves.

\section{Statistical Methods}

Data analysis was carried out using R (Version 3.4) [44] and the methods provided by the GAMLSS package [45]. Plausibility was tested for all data. Beyond that, no outliers were excluded. Age- and sex-related percentile curves for the particular skinfolds were estimated assuming an underlying four-parameter Box-Cox power exponential distribution [46] as an extension of the LMS method [47]. As LIFE Child recruits whole families with more than one child and has a longitudinal study design, our data contain measurements for siblings and also multiple measurements from individual children. For this reason, a resampling method was used to allow the inclusion of all measurements in the modelling of reference curves [48, 49]. Percentile curves were calculated for the 3rd (P3), 10th (P10), 50th (M, median), 90th (P90) and 97th (P97) percentiles. Model quality was controlled using worm plots [50].

\section{Results}

\section{Participants}

The reference population comprised young people from 1,710 families, producing a total of 6,344 cases (46.9\% female cases) (of complete ST, HC and WC measurements), which provided the basis for determining percentiles. The cutoffs for defining underweight, overweight and obesity were the 3rd, 90th and 97th percentiles of the age- and sex-adjusted BMI distribution $[13,51]$, respectively. In $5.5 \%$ of cases, the participants were overweight $(169$ female, 182 male), in 2.8\% they were obese (92 female, 90 male) and in $8.7 \%$, they were underweight (253 female, 301 male). In $82.6 \%$ of cases $(5,254)$, the children and adolescents were normal weight. The mean BMI-SDS was -0.036 among girls and -0.073 among boys. The mean age was 9.97 years for girls and 9.84 years for boys. The socioeconomic status of $10.0 \%$ 
Rönnecke et al.: Age- and Sex-Related Percentiles of Skinfold Thickness, Waist and Hip Circumference, Waist-to-Hip Ratio and Waist-to-Height Ratio: Results from a

Population-Based Pediatric Cohort in Germany (LIFE Child)

of the families was classified as low level, of $48.2 \%$ as middle level, and of $41.8 \%$ as high level according to the Winkler index. There are missing values for BMI $(n=3)$ and socioeconomic status $(n=105)$.

\section{Percentiles of ST, WC, HC, WHR, and WHtR in a Healthy Cohort}

Age- and sex-related smoothed percentile curves for each skinfold are shown in Figure 1; percentile curves for the waist and hip parameters are represented in Figure 2. The tables can be found in the supplementary material. For each gender, the reference intervals are represented in online supplementary tables 1-16 (available at http://content.karger.com/ ProdukteDB/produkte.asp?doi $=494767$ ) as the values of the 3rd, 10th, 90th and 97th percentiles in addition to the median ( $M=50$ th percentile). The tables also contain the following parameters, which describe the age-based distribution: sigma ( $\sigma$, scale), nu ( $v$, skewness), and tau ( $\tau$, kurtosis, only for ST). The median triceps ST percentile (M) shows a decrease in boys and girls starting at the age of 3 . In boys, the values increase from the age of 7 until they reach a peak at 12 years with a subsequent decline. In girls, we see increasing values from 6 years upwards until the age of 16. Girls have higher ST than boys from 4 years upwards, and the difference increases during adolescence (12-16 years), an effect mainly induced by the reduced ST in boys. We found similar trends regarding the biceps ST curves: there is a decrease in both sexes until the age of 6 years for girls, and until 7 years for boys. From this point on, we see ST rising in both sexes, but again with the boys' measurement peaking at the age of 12 in contrast to the girls' values. We see higher values among girls than we do among boys of the same age, across the whole age range. From the age of 3-6, there is, again, a decrease in the percentile curves for subscapular skinfold. In contrast to the triceps and biceps median percentile curves, this one shows a continuous increase for boys of this age except in the most extreme percentile (P97). Girls' subscapular ST is higher than the boys' values in all but the 90th and 97th curves. Among boys, the values for the 97th percentile in particular show a large increase in adolescence. Female iliac crest ST percentiles follow a continuous upward trend with increasing age, whereas male percentiles decrease up to the age of 5.5 with a subsequent increase. Iliac crest ST values for most percentile curves among the girls are higher than those for the boys. The 97th percentile is an exception for boys in adolescence: from the age of 10.5 it exceeds the values of the girls, reaching its peak at the age of 13.5 followed by a decrease. Overall, we see two different patterns in the trends of the ST percentile curves: in the biceps and triceps ST, the boys' values peak at the beginning of adolescence, with a subsequent decrease, while girls' percentiles rise across the age range. Among the subscapular and iliac crest ST values, there is no peak in the boys' median values (P50) but the curves all rise with increasing age, with particularly high values for P90 and P97. All percentiles (except the iliac crest curves for the girls) decrease during preschool age and increase from the age of approximately 6 years.

Female and male median HC percentiles (M) increase starting at the age of 3. While the boys' median percentile shows a continuous increase, the girls' median percentile levels slightly at the age of 16 . All percentile curves show similar trends in both sexes, but the values for girls are higher overall. We found similar trends regarding the WC percentile curves: we see a relatively constant increase of the median percentiles from 3 to 16 years in both sexes with a plateau at the age of 15.5 for the girls. Overall, the 97 th percentiles show a large increase in adolescence and boys show higher values in all WC percentile curves with an increasing difference between both sexes. Both female and male WHR percentiles follow a downward trend with increasing age. We found a slight increase in WHR percentiles at the age of 16 for the boys in contrast to the girls' values. Values for boys are higher than those for girls of the same age across the age range and we see an increasing difference due to the increase in the boys' values towards the age of 16 . The median WHtR percentiles show a decrease in both 
Rönnecke et al.: Age- and Sex-Related Percentiles of Skinfold Thickness, Waist and Hip Circumference, Waist-to-Hip Ratio and Waist-to-Height Ratio: Results from a

Population-Based Pediatric Cohort in Germany (LIFE Child)

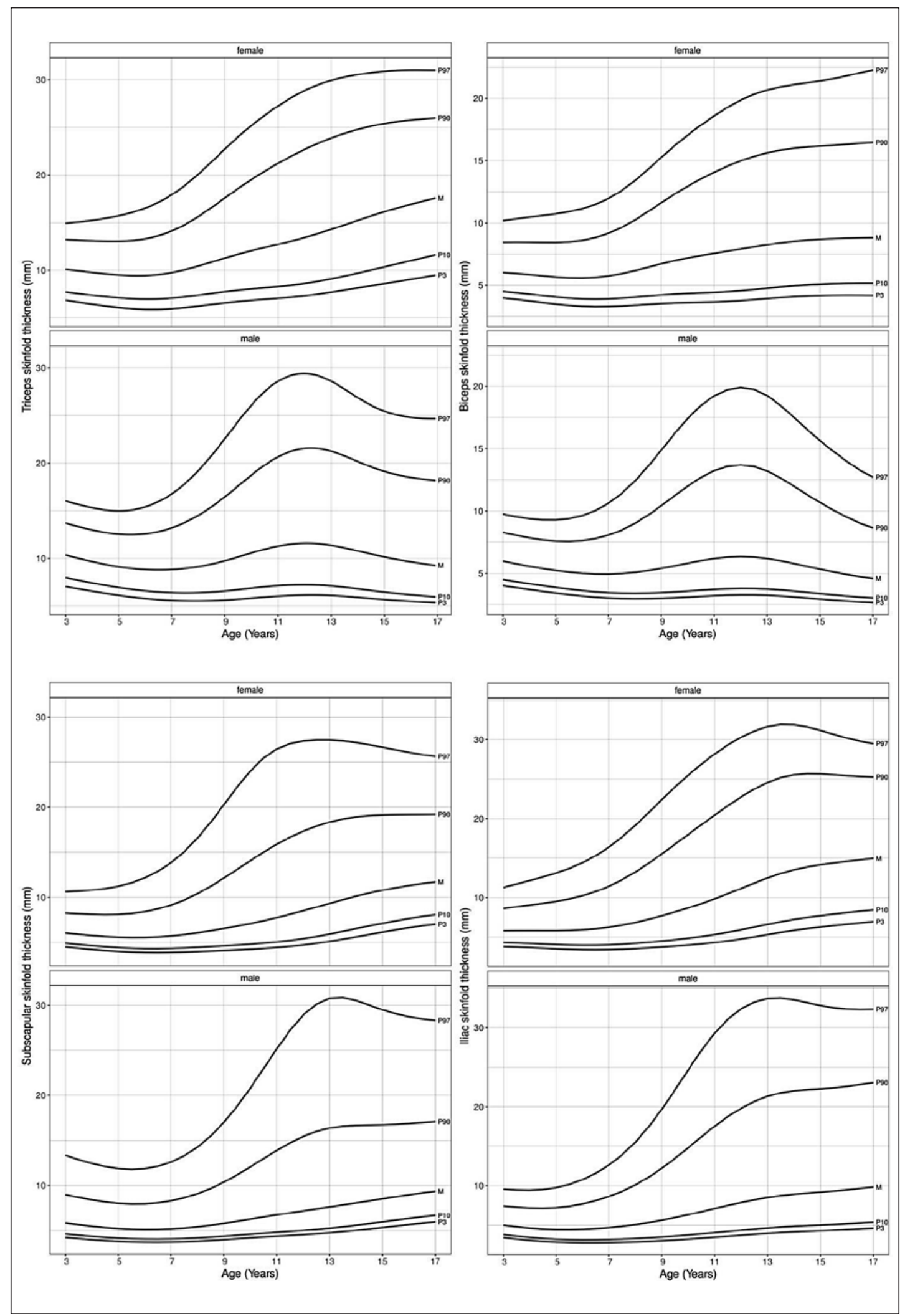

Fig. 1. Smoothed percentile curves for triceps, biceps, subscapular an iliac crest skinfold thickness (mm) in females/males aged 3-16, based on the reference population of the LIFE-Child Cohort (total: $n=6,344$ ). Curves included are for 3rd (P3), 10th (P10), 50th/median (M), 90th (P90) and 97th (P97) percentiles. 
Rönnecke et al.: Age- and Sex-Related Percentiles of Skinfold Thickness, Waist and Hip Circumference, Waist-to-Hip Ratio and Waist-to-Height Ratio: Results from a

Population-Based Pediatric Cohort in Germany (LIFE Child)
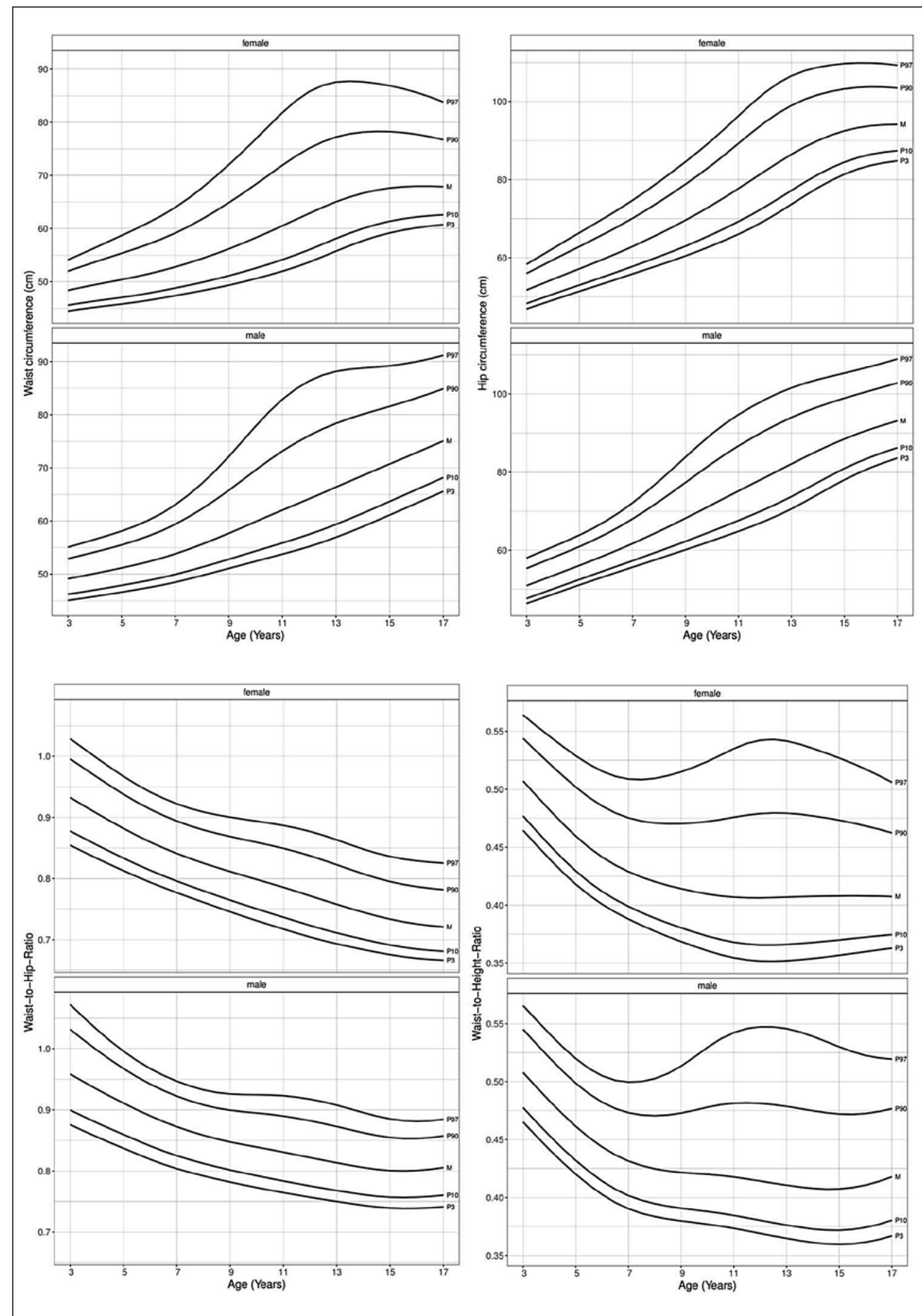

Fig. 2. Smoothed percentile curves for hip circumference $(\mathrm{cm})$, waist circumference $(\mathrm{cm})$, waist-to-hip ratio and waist-to-height ratio in females/males aged 3-16, based on the reference population of the LIFE-Child cohort (total: $n=6,344)$. Curves included are for 3rd (P3), 10th (P10), 50th/median (M), 90th (P90) and 97th (P97) percentiles. 
Rönnecke et al.: Age- and Sex-Related Percentiles of Skinfold Thickness, Waist and Hip Circumference, Waist-to-Hip Ratio and Waist-to-Height Ratio: Results from a

Population-Based Pediatric Cohort in Germany (LIFE Child)

sexes with a plateau at the age of 12.5 for the girls and increasing values at the age of 15.5 for the boys. The 97 th percentile of both sexes differs most significantly from these trends: after a decrease, we see increasing values from 8 years up to the age of 13 , followed by a decline. All WHtR percentiles show slightly higher values for boys.

\section{Discussion}

In this paper, ST, WC, HC, WHR and WHtR values are used as a measurement of BFP and body fat. As we see different trends for the specific anthropometric data, the combination of different measures provides more information compared to the use of BMI as a sole parameter for overweight or obesity. The subscapular and iliac crest skinfolds, localized at the trunk of the body, show continuously increasing values in line with the increasing BMI during childhood and adolescence. The continuous increase of HC and WC as dimensions of trunk body fat fits these increasing ST values, especially during adolescence. In contrast, the two skinfold values measured on the arm close to defined muscles show a peak for boys. This can be explained by the testosterone-mediated muscle growth during puberty [52]. Using Slaughter et al.'s BFP equations, it is possible to estimate the BFP for the different age- and gender-related percentiles. According to these estimates, BFP of the median percentile is the same at the age of 3 for both sexes (approximately 15.7\%). At the age of 6, girls show a slightly higher BFP of $14.5 \%$ compared to the boy's median percentile $(13.6 \%)$. This difference increases up to the age of 16 (girls: $25.0 \%$, boys: $14.2 \%$ ) in spite of the peak in the male triceps SF values at the age of 12 (girls: $20.4 \%$, boys: $16.5 \%$ ). Schwandt et al. [25] calculated the BFP of children in Nuremberg aged 3 to 18 years. Their calculations show a higher median BFP in females than in boys and a peak for boys at the age of 11 , which is in line with our results. In comparison with their data, our values are higher overall. Among participants in the 97th percentile for triceps and subscapular ST, we estimated a BFP of up to $54.6 \%$ for girls and $48.1 \%$ for boys, which are much higher values than those presented by Schwandt et al. [25].

Higher values for girls are a feature of HC and all ST percentiles; WC percentiles are an exception with higher values for boys. Since the HC is influenced by bone structure, gluteal muscle mass and the subcutaneous fat tissue, it is rarely used on its own [53]. Rather it tends to feature in combination with other parameters such as WC or height: we see higher values of WHR for boys across the whole age range and an increasing difference between the sexes. This is due to the increasing values among boys at the age of 16 in contrast to the still decreasing values in girls. In total, the median WHR shows a decrease of $16.3 \%$ for boys and $22.5 \%$ for girls. This higher figure for girls is due to the increasing HC and formation of the waist during puberty [54]. Different studies note critically that a reduction of body weight is associated with a reduction of both WC and HC. As such, the WHR does not change despite slimmer body proportions $[26,55]$. However, WHtR shows gender-specific trends: in girls it levels off at the age of 12.5; in boys we see an increase at the age of 15.5 with slightly higher values at 16.5 compared to girls. These results may be influenced by the difference in growth patterns, with girls reaching their final height around 16 and boys reaching theirs around 18 [26].The respective trends for skinfold percentiles have also been a feature in other comparable studies of German cohorts. The KiGGS study [56] provides data for triceps and subscapular ST of healthy children aged 3 to 18 years measured at different examination centers all over Germany. Their results also show higher ST for female subjects in general as well as similar trends of percentile curves including the peak of boy's triceps ST at the age of 12. Over all, the ST percentiles of the KiGGS study, especially those of the triceps, are higher than those in our study. But the boy's subscapular ST percentiles show lower values for the 90th and 97th percentile up to the age of 6-7 years and from 12-13 years on (Fig. 3). Our 
Rönnecke et al.: Age- and Sex-Related Percentiles of Skinfold Thickness, Waist and Hip Circumference, Waist-to-Hip Ratio and Waist-to-Height Ratio: Results from a

Population-Based Pediatric Cohort in Germany (LIFE Child)

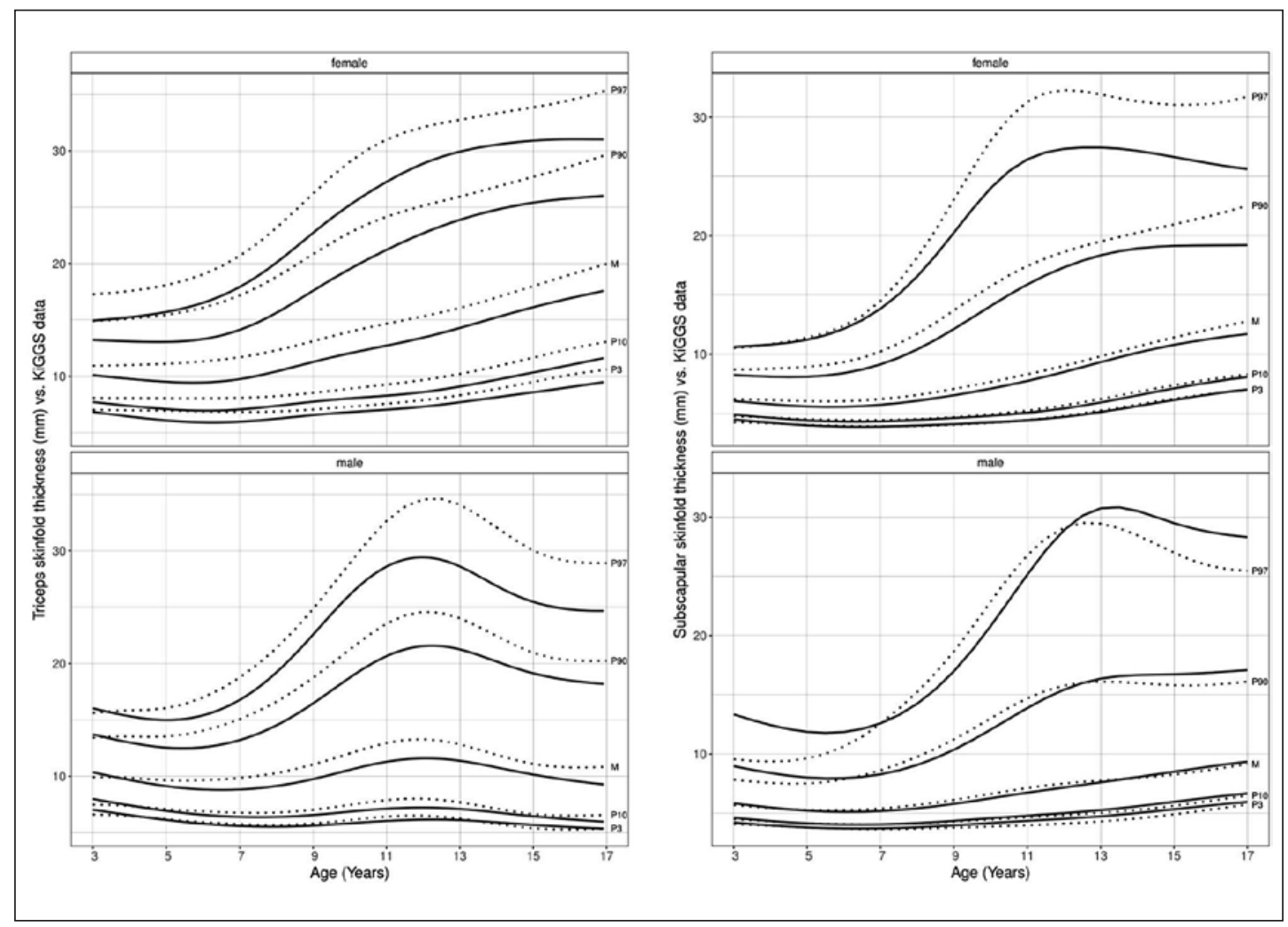

Fig. 3. Smoothed percentile curves for triceps and subscapular skinfold thickness ( $\mathrm{mm}$ ) in females/males aged 3-16, based on the reference population of the LIFE-Child Cohort (total: $n=6,344$ ) in comparison with the KiGGS data (dotted lone). Curves included are for 3rd (P3), 10th (P10), 50th/median (M), 90th (P90) and 97th (P967).

results for ST also are comparable to those of international studies, such as recent data from Canada [56] and Norway [57]. Only in Chinese children, the ST values seem to be much higher than those from similar cohorts, which underlines the role of ethnicity [16]. The KiGGS study provides data for $\mathrm{HC}, \mathrm{WC}, \mathrm{WHR}$, and WHtR in children aged 11-18 years. Our data therefore provide additional information about development during childhood. The KiGGS' HC percentiles show a very similar course to ours, but its values for P90 and P97 are higher, probably due to the BMI distribution across the participants. Furthermore, the KiGGS' percentiles for WC show higher values for all curves, but different trends for P90 and P97, with an increase at the age of 13.5 in girls, in contrast to the decline revealed in our data. Our trends for WHR are very similar to the KiGGS' percentiles, though again with higher values for all KiGGS percentiles and more pronounced differences for WHtR percentiles: we see much higher WHtR values, in particular, at the beginning and end of the age range of the KiGGS' cohort (Fig. 4). In comparison to data for adolescents aged 12-18 years from the Bavarian Family Heart Study [20] our circumference values are lower overall. Waist and hip parameters are also comparable to those of international studies from Canada [56], Great Britain [58], and Brazil [59]. WC and WHtR in Greek adolescents [16] and WC in Chinese children seem to be higher than in our participants [60]. In general, not many studies include preschool children; therefore, our circumference data provide additional important and recent information. Several percentile curves show interesting, if subtle, trends at the end of the studied age range, for example the plateaus in female WC and HC percentiles in contrast to the increasing 
Rönnecke et al.: Age- and Sex-Related Percentiles of Skinfold Thickness, Waist and Hip Circumference, Waist-to-Hip Ratio and Waist-to-Height Ratio: Results from a
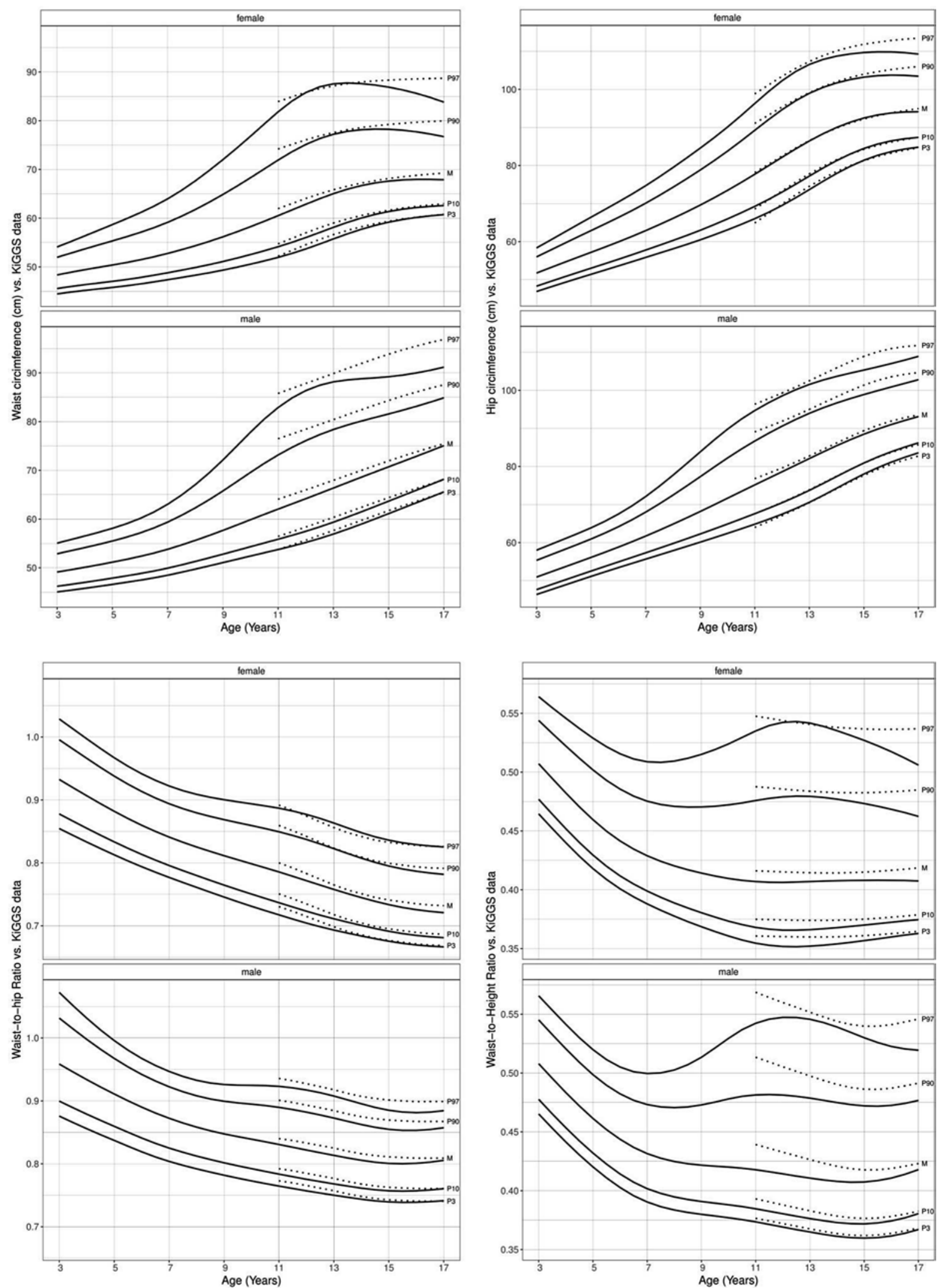

(For legend see next page.) 
Rönnecke et al.: Age- and Sex-Related Percentiles of Skinfold Thickness, Waist and Hip Circumference, Waist-to-Hip Ratio and Waist-to-Height Ratio: Results from a

Population-Based Pediatric Cohort in Germany (LIFE Child)

male WC and HC percentiles or the slight increase in boys' WHR and WHtR percentiles at the age of 16 . It will be interesting to explore these developments in upcoming analyses.

Looking at the parameters separately, we see differences in the prevalence of values > 97th percentile: $2.4 \%$ of the examined cases have WC values $>97$ th percentile compared to $2.8 \%$ with values $>97$ th percentile for BMI. The prevalence of cases with WHR or values for the sum of triceps and subscapular ST $>97$ th percentile (2.2 and $2.4 \%$ respectively) is lower than for BMI. In our cohort, the percentage of cases of overweight, defined by the cutoff of the 90th percentile of BMI, was $5.5 \%$ compared to $6.6 \%$ of cases $>90$ th percentile value for the sum of triceps and subscapular ST. 5.8\% of the WHR values and $5.4 \%$ of the WC values of the examined cases are located above the cutoff of the 90th percentile. The greater prevalence of $>90$ th percentile cases for the sum of triceps and subscapular ST, in comparison with >90th percentile values for BMI, can largely be attributed to the enrichment of the sample group for the first measure with male cases. Thus, ST measurement may be a helpful examination for identifying subcutaneous fat tissue and high BFP in normal-weight boys.

Overall, our results are consistent with comparable studies of healthy German children. One limitation of our results may be the underrepresentation of overweight and obese children compared to their prevalence in the wider German population [61]. As the complete LIFE Child study contains a subcohort enriched with overweight and obese children, we tested an inclusion of these cases in our calculations. But while this leads to an enrichment in the obese sector of to $9.9 \%$, it only raises the quota of overweight cases to $7.3 \%$. As such, we decided not to include this subcohort, because of the unbalanced enrichment and level of measurement inaccuracy, which is particularly high in these sectors [62]. As high BMI levels are associated with low socioeconomic status [2], our results could be affected directly and indirectly by the distribution of social status in our cohort with an enrichment in middle and high social levels compared to reference cohorts such as KiGGS (27.8\% low, 45.3\% middle, $25.1 \%$ high social level) [41,63]. As with the wider population of Leipzig, the majority of the young participants were Caucasian. This may limit the comparability of our data to those of other countries, because both socioeconomic status and ethnic differences influence overweight and obesity prevalence rates [64]. Furthermore, a general problem in dealing with ST is measuring inaccuracy, especially in overweight individuals [62]. We minimized this inaccuracy by using the median of 3 measurements for each skinfold, and having skilled investigators perform the examinations using standardized procedures and the same caliper models. The main advantages of our study design are the relatively large age range, the standardized examination setting, the large number of participants and, especially for upcoming analyses, the longitudinal character of the project, which offers the opportunity to observe developments in the anthropometric data of single individuals over time.

\section{Conclusion}

Our analysis has produced current percentile curves for biceps, triceps, subscapular and iliac ST, and WC, HC, WHR and WHtR in children and adolescents. These curves have been defined by modern statistical methods and can be used to identify abdominal obesity in young people. We found gender-related differences in distribution of fat tissue and trends in fat

Fig. 4. Smoothed percentile curves for hip circumference $(\mathrm{cm})$, waist circumference $(\mathrm{cm})$, waist-to-hip ratio and waist-to-height ratio in females/males aged 3-16, based on the reference population of the LIFE-Child Cohort (total: $n=6.344$ ) in comparison with the KiGGS' data (dotted lines). Curves included are for 3rd (P3), 10th (P10), 50th/median (M), 90th (P90) and 97th (P97) percentiles. 
Rönnecke et al.: Age- and Sex-Related Percentiles of Skinfold Thickness, Waist and Hip Circumference, Waist-to-Hip Ratio and Waist-to-Height Ratio: Results from a

Population-Based Pediatric Cohort in Germany (LIFE Child)

tissue quantity during childhood and puberty, which in part can be attributed to differences in muscle growth and growth patterns. We even witness gender-related differences before puberty, such as higher WC values among boys, higher values for ST and HC in girls, or the different iliac crest ST trends for preschool children. Due to the shortage of (German) studies investigating these parameters, our data are among the most current results available [65]. In particular, this is the first study to present age- and sex-specific circumference data for the whole age range of 3-16 years for children in Germany. The longitudinal character of LIFE Child will provide information about development in individuals in the years ahead.

\section{Acknowledgment}

The results presented here are part of the LIFE Child study. We wish to thank everybody in the team performing the examinations, and of course, the children who participated in the study and their parents for their collaboration. We acknowledge support from the German Research Foundation (DFG) and Universität Leipzig within the program of Open Access Publishing.

\section{Funding}

This publication is supported by LIFE - Leipzig Research Center for Civilization Diseases, University of Leipzig. LIFE is funded by the European Union via the European Social Fund (ESF), by the European Regional Development Fund (ERDF), and by the Free State of Saxony within the framework of the excellence initiative of the Saxonian Ministry of Science and Arts (SMWK), Free State of Saxony, Germany.

\section{Statement of Ethics}

The study was designed pursuant to the declaration of Helsinki and was approved by the Ethical Committee of the University of Leipzig (reference number: Reg. No. 264-1019042010). LIFE-Child is registered by the trial number: NCT02550236. Parental consent was required for all examinations, plus the participant's own consent if aged 12 or over.

\section{Author Contributions}

W. Kiess, E. Rönnecke, and M. Vogel designed the study, A. Jurkutat, M. Schlingmann, S. Bußler, and N. Grafe gathered the data, M. Vogel and E. Rönnecke analyzed the data. W. Kiess, M. Vogel, and A. Körner vouch for the data and the analyses. E. Rönnecke wrote the paper. W. Kiess and A. Körner decided, and all authors agreed to publish the paper.

There were no agreements concerning confidentiality of the data between the sponsor and the authors or the institutions named in the credit lines.

\section{Disclosure Statement}

The authors confirm that there is no conflict of interest. 
Rönnecke et al.: Age- and Sex-Related Percentiles of Skinfold Thickness, Waist and Hip Circumference, Waist-to-Hip Ratio and Waist-to-Height Ratio: Results from a Population-Based Pediatric Cohort in Germany (LIFE Child)

\section{References}

1 Robert Koch-Institut. Übergewicht und Adipositas im Kindes- und Jugendalter in Deutschland - Querschnittergebnisse aus KiGGS Welle 2 und Trends. RKI-Bib1. Berlin, Robert Koch-Institut, 2018.

2 Kurth BM, Schaffrath Rosario A. [The prevalence of overweight and obese children and adolescents living in Germany. Results of the German Health Interview and Examination Survey for Children and Adolescents (KiGGS)]. Bundesgesundheitsbl Gesundheitsforsch Gesundheitsschutz. 2007 May-Jun;50(5-6):736-43.

3 Parsons TJ, Power C, Logan S, Summerbell CD. Childhood predictors of adult obesity: a systematic review. Int J Obes Relat Metab Disord. 1999 Nov;23 Suppl 8:S1-107.

4 Kelsey MM, Zaepfel A, Bjornstad P, Nadeau KJ. Age-related consequences of childhood obesity. Gerontology. 2014;60(3):222-8.

5 Wahl S, Yu Z, Kleber M, Singmann P, Holzapfel C, He Y, et al. Childhood obesity is associated with changes in the serum metabolite profile. Obes Facts. 2012;5(5):660-70.

6 Juonala M, Raitakari M, S A Viikari J, Raitakari OT. Obesity in youth is not an independent predictor of carotid IMT in adulthood. The Cardiovascular Risk in Young Finns Study. Atherosclerosis. 2006 Apr;185(2):388-93.

7 Freedman DS, Ogden CL, Kit BK. Interrelationships between BMI, skinfold thicknesses, percent body fat, and cardiovascular disease risk factors among U.S. children and adolescents. BMC Pediatr. 2015 Nov;15(1):188.

8 Kalantari S, Khalili D, Asgari S, Fahimfar N, Hadaegh F, Tohidi M, et al. Predictors of early adulthood hypertension during adolescence: a population-based cohort study. BMC Public Health. 2017 Nov;17(1):915.

9 Baker JL, Olsen LW, Sørensen TI. Childhood body-mass index and the risk of coronary heart disease in adulthood. N Engl J Med. 2007 Dec;357(23):2329-37.

10 DAG - Deutsche Adipositas Gesellschaft. Leitlinien. http://www.adipositas-gesellschaft.de/index.php?id=9. Accessed 02 Dec 2015.

11 Himes JH, Dietz WH; The Expert Committee on Clinical Guidelines for Overweight in Adolescent Preventive Services. Guidelines for overweight in adolescent preventive services: recommendations from an expert committee. Am J Clin Nutr. 1994 Feb;59(2):307-16.

12 Bohn B, Müller MJ, Simic-Schleicher G, Kiess W, Siegfried W, Oelert M, et al.; APV Initiative and the German BMBF Competence Network Obesity. BMI or BIA: Is Body Mass Index or Body Fat Mass a Better Predictor of Cardiovascular Risk in Overweight or Obese Children and Adolescents? A German/Austrian/Swiss Multicenter APV Analysis of 3,327 Children and Adolescents. Obes Facts. 2015;8(2):156-65.

13 Kromeyer-Hauschild K, Wabitsch M, Kunze D, Geller F, Geiß HC, Hesse V, et al. Perzentile für den Body-MassIndex für das Kindes- und Jugendalter unter Heranziehung verschiedener deutscher Stichproben. Monatsschr Kinderheilkd. 2001;149(8):807-18.

14 Plachta-Danielzik S, Gehrke MI, Kehden B, Kromeyer-Hauschild K, Grillenberger M, Willhöft C, et al. Body fat percentiles for German children and adolescents. Obes Facts. 2012;5(1):77-90.

15 Wells JC. Sexual dimorphism of body composition. Best Pract Res Clin Endocrinol Metab. 2007 Sep;21(3): 415-30.

16 Lee RL, Lee PH, Sze DM, Chien WT. Anthropometric profile of Hong Kong children and adolescents: the Wellness Population of Youth Study. J Am Soc Hypertens. 2017 Apr;11(4):196-203.e4.

17 Becker C. Formulierung populationsspezifischer Algorithmen zur Bestimmung der Körperfettmasse von Kindern, Jugendlichen und Erwachsenen, basierend auf Feldmethoden mit Air Displacement Plethysmography als Referenz: Dissertation zur Erlangung des Doktorgrades der Agrar- und Ernährungswissenschaftlichen Fakultät der Christian-Albrechts-Universität zu Kiel, Christian-Albrechts-Universität zu Kiel, 2002.

18 ChiriŢă-Emandi A, Papa MC, Abrudan L, Dobrescu MA, Puiu M, Velea IP, et al. A novel method for measuring subcutaneous adipose tissue using ultrasound in children - interobserver consistency. Rom J Morphol Embryol. 2017;58(1):115-23.

19 Himes JH. Challenges of accurately measuring and using BMI and other indicators of obesity in children. Pediatrics. 2009 Sep;124 Suppl 1:S3-22.

20 Haas GM, Liepold E, Schwandt P. Percentile curves for fat patterning in German adolescents. World J Pediatr. $2011 \mathrm{Feb} ; 7(1): 16-23$.

21 Nooyens AC, Koppes LL, Visscher TL, Twisk JW, Kemper HC, Schuit AJ, et al. Adolescent skinfold thickness is a better predictor of high body fatness in adults than is body mass index: the Amsterdam Growth and Health Longitudinal Study. Am J Clin Nutr. 2007 Jun;85(6):1533-9.

22 Zimmermann MB, Gübeli C, Püntener C, Molinari L. Detection of overweight and obesity in a national sample of 6-12-y-old Swiss children: accuracy and validity of reference values for body mass index from the US Centers for Disease Control and Prevention and the International Obesity Task Force. Am J Clin Nutr. 2004 May; 79(5):838-43.

23 Kromeyer-Hauschild K, Glässer N, Zellner K. Percentile curves for skinfold thickness in 7- to 14-year-old children and adolescents from Jena, Germany. Eur J Clin Nutr. 2012 May;66(5):613-21.

24 Weinand C, Müller S, Zabransky S, Danker-Hopfe H. [Saarland Growth Study: analyses of body composition of children, aged 3 to 11 years. Measurement of height, weight, girth (abdomen, upper arm, calf) and skinfolds (triceps, biceps, subscapular,suprailiacal, abdominal) and bioelectric impedance (BIA)]. Wien Med Wochenschr. 2000;150(7):140-4. 
Rönnecke et al.: Age- and Sex-Related Percentiles of Skinfold Thickness, Waist and Hip Circumference, Waist-to-Hip Ratio and Waist-to-Height Ratio: Results from a

Population-Based Pediatric Cohort in Germany (LIFE Child)

25 Schwandt P, von Eckardstein A, Haas GM. Percentiles of percentage body fat in german children and adolescents: an international comparison. Int J Prev Med. 2012 Dec;3(12):846-52.

26 Neuhauser H, Schienkiewitz A, Schaffrath Rosario A, et al. (2013) Beiträge zur Gesundheitsberichterstattung des Bundes: Referenzperzentile für anthropometrische Maßzahlen und Blutdruck aus der Studie zur Gesundheit von Kindern und Jugendlichen in Deutschland (KiGGS), 2. erweiterte Auflage, Berlin

27 Fu J, Hofker M, Wijmenga C. Apple or pear: size and shape matter. Cell Metab. 2015 Apr;21(4):507-8.

28 Hübers M, Pourhassan M, Braun W, Geisler C, Müller MJ. Definition of new cut-offs of BMI and waist circumference based on body composition and insulin resistance: differences between children, adolescents and adults. Obes Sci Pract. 2017 Aug;3(3):272-81.

29 Bravo J, Raimundo AM, Santos DA, Timón R, Sardinha LB. Abdominal obesity in adolescents: development of age-specific waist circumference cut-offs linked to adult IDF criteria. Am J Hum Biol. 2017 Nov;29(6):29.

30 Haas GM, Liepold E, Schwandt P. Predicting Cardiovascular Risk Factors by dIfferent Body Fat Patterns in 3850 German Children: the PEP Family Heart Study. Int J Prev Med. 2011 Jan;2(1):15-9.

31 Goran MI, Gower BA, Treuth M, Nagy TR. Prediction of intra-abdominal and subcutaneous abdominal adipose tissue in healthy pre-pubertal children. Int J Obes Relat Metab Disord. 1998 Jun;22(6):549-58.

32 Sangi H, Mueller WH. Which measure of body fat distribution is best for epidemiologic research among adolescents? Am J Epidemiol. 1991 May;133(9):870-83.

33 Lichtenauer M, Wheatley SD, Martyn-St James M, Duncan MJ, Cobayashi F, Berg G, et al. Efficacy of anthropometric measures for identifying cardiovascular disease risk in adolescents: review and meta-analysis. Minerva Pediatr. 2018 Aug;70(4):371-82.

34 Janssen I, Katzmarzyk PT, Ross R. Waist circumference and not body mass index explains obesity-related health risk. Am J Clin Nutr. 2004 Mar;79(3):379-84.

35 McCarthy HD, Ellis SM, Cole TJ. Central overweight and obesity in British youth aged 11-16 years: cross sectional surveys of waist circumference. BMJ. 2003 Mar;326(7390):624.

36 Savva SC, Tornaritis M, Savva ME, Kourides Y, Panagi A, Silikiotou N, et al. Waist circumference and waist-toheight ratio are better predictors of cardiovascular disease risk factors in children than body mass index. Int J Obes Relat Metab Disord. 2000 Nov;24(11):1453-8.

37 Taylor SA, Hergenroeder AC. Waist circumference predicts increased cardiometabolic risk in normal weight adolescent males. Int J Pediatr Obes. 2011 Jun;6(2-2):e307-11.

38 Freedman DS, Serdula MK, Srinivasan SR, Berenson GS. Relation of circumferences and skinfold thicknesses to lipid and insulin concentrations in children and adolescents: the Bogalusa Heart Study. Am J Clin Nutr. 1999 Feb;69(2):308-17.

39 Blüher S, Molz E, Wiegand S, Otto KP, Sergeyev E, Tuschy S, et al.; Adiposity Patients Registry Initiative and German Competence Net Obesity. Body mass index, waist circumference, and waist-to-height ratio as predictors of cardiometabolic risk in childhood obesity depending on pubertal development. J Clin Endocrinol Metab. 2013 Aug;98(8):3384-93.

40 Quante M, Hesse M, Döhnert M, Fuchs M, Hirsch C, Sergeyev E, et al.; LIFE Child Study Investigators. The LIFE child study: a life course approach to disease and health. BMC Public Health. 2012 Nov;12(1):1021.

41 Poulain T, Baber R, Vogel M, Pietzner D, Kirsten T, Jurkutat A, et al.; LIFE Child study team. The LIFE Child study: a population-based perinatal and pediatric cohort in Germany. Eur J Epidemiol. 2017 Feb;32(2):14558.

42 Winkler J, Stolzenberg H. [Social class index in the Federal Health Survey]. Gesundheitswesen. 1999 Dec; 61(Spec No):S178-83.

43 Slaughter MH, Lohman TG, Boileau RA, Horswill CA, Stillman RJ, Van Loan MD, et al. Skinfold equations for estimation of body fatness in children and youth. Hum Biol. 1988 Oct;60(5):709-23.

44 R Core Team. R: A language and environment for statistical. Vienna, Austria: R Foundation for Statistical Computing; 2014.

45 Rigby RA, Stasinopoulos DM. Automatic smoothing parameter selection in GAMLSS with an application to centile estimation. Stat Methods Med Res. 2014 Aug;23(4):318-32.

46 Rigby RA, Stasinopoulos DM. Smooth centile curves for skew and kurtotic data modelled using the Box-Cox power exponential distribution. Stat Med. 2004 Oct;23(19):3053-76.

47 Cole TJ, Green PJ. Smoothing reference centile curves: the LMS method and penalized likelihood. Stat Med. 1992 Jul;11(10):1305-19.

48 Vogel M, Kirsten T, Kratzsch J, Engel C, Kiess W. A combined approach to generate laboratory reference intervals using unbalanced longitudinal data. J Pediatr Endocrinol Metab. 2017 Jul;30(7):767-73.

49 Dathan-Stumpf A, Vogel M, Hiemisch A, Thiery J, Burkhardt R, Kratzsch J, et al. Pediatric reference data of serum lipids and prevalence of dyslipidemia: results from a population-based cohort in Germany. Clin Biochem. 2016 Jul;49(10-11):740-9.

50 van Buuren S, Fredriks M. Worm plot: a simple diagnostic device for modelling growth reference curves. Stat Med. 2001 Apr;20(8):1259-77.

51 Wabitsch M, Kunze D. Konsensbasierte (S2) Leitlinie zur Diagnostik, Therapie und Prävention von Übergewicht und Adipositas im Kindes- und Jugendalter: Version 15.10.2015. www.a-g-a.de.

52 Hou WW, Tse MA, Lam TH, Leung GM, Schooling CM. Adolescent testosterone, muscle mass and glucose metabolism: evidence from the 'Children of 1997' birth cohort in Hong Kong. Diabet Med. 2015 Apr;32(4):505-12. 
Rönnecke et al.: Age- and Sex-Related Percentiles of Skinfold Thickness, Waist and Hip Circumference, Waist-to-Hip Ratio and Waist-to-Height Ratio: Results from a

Population-Based Pediatric Cohort in Germany (LIFE Child)

53 Seidell JC, Pérusse L, Després JP, Bouchard C. Waist and hip circumferences have independent and opposite effects on cardiovascular disease risk factors: the Quebec Family Study. Am J Clin Nutr. 2001 Sep;74(3):31521.

54 de Ridder CM, de Boer RW, Seidell JC, Nieuwenhoff CM, Jeneson JA, Bakker CJ, et al. Body fat distribution in pubertal girls quantified by magnetic resonance imaging. Int J Obes Relat Metab Disord. 1992 Jun;16(6): 443-9.

55 Daniels SR, Khoury PR, Morrison JA. Utility of different measures of body fat distribution in children and adolescents. Am J Epidemiol. 2000 Dec;152(12):1179-84.

56 Kuhle S, Ashley-Martin J, Maguire B, Hamilton DC. Percentile curves for skinfold thickness for Canadian children and youth. Peer J. 2016 Jul;4:e2247.

57 Brannsether B, Roelants M, Bjerknes R, Júlíusson PB. New reference charts for weight-related body measurements in children. Tidsskr Nor Laegeforen. 2016 Nov;136(21):1828-30.

58 McCarthy HD, Jarrett KV, Emmett PM, Rogers I. Trends in waist circumferences in young British children: a comparative study. Int J Obes. 2005 Feb;29(2):157-62.

59 Frignani RR, Passos MA, Ferrari GL, Niskier SR, Fisberg M, Cintra IP. Reference curves of the body fat index in adolescents and their association with anthropometric variables. J Pediatr (Rio J). 2015 May-Jun;91(3):24855.

60 Bacopoulou F, Efthymiou V, Landis G, Rentoumis A, Chrousos GP. Waist circumference, waist-to-hip ratio and waist-to-height ratio reference percentiles for abdominal obesity among Greek adolescents. BMC Pediatr. 2015 May;15(1):50.

61 Blüher S, Meigen C, Gausche R, Keller E, Pfäffle R, Sabin M, et al. Age-specific stabilization in obesity prevalence in German children: a cross-sectional study from 1999 to 2008. Int J Pediatr Obes. 2011 Jun;6(2-2):e199-206.

62 Kriemler S, Puder J, Zahner L, Roth R, Meyer U, Bedogni G. Estimation of percentage body fat in 6- to 13-year-old children by skinfold thickness, body mass index and waist circumference. Br J Nutr. 2010 Nov; 104(10):156572.

63 Winkler J, Stolzenberg H. Adjustierung des Sozialen-Schicht-Index für die Anwendung im Kinder-und Jugendgesundheitssurvey (KiGGS). Wismarer Diskussionspapiere. Wismar, Hochschule Wismar, Fakultät für Wirtschaftswissenschaften, 2009.

64 Ujcic-Voortman JK, Bos G, Baan CA, Verhoeff AP, Seidell JC. Obesity and body fat distribution: ethnic differences and the role of socio-economic status. Obes Facts. 2011;4(1):53-60.

65 Schwandt P, Kelishadi R, Haas GM. First reference curves of waist circumference for German children in comparison to international values: the PEP Family Heart Study. World J Pediatr. 2008 Nov;4(4):259-66. 